\title{
A New Rang-instantaneous Doppler ISAR Imaging Algorithm Baoping Wang ${ }^{1,}$, , Chao Sun ${ }^{1, b}$, Junjie Guo ${ }^{1, c}$ \\ ${ }^{1}$ Science and Technology on UAV Laboratory, Northwestern Polytechnical University, Xi'an 710065, China \\ awbpluo@sina.com, bwo_sunchao1987@sina.com, cguojunjie19871212@163.com
}

\begin{abstract}
Keywords: ISAR; sparse representation; time-frequency transform; time-frequency basis
\end{abstract}
\begin{abstract}
ISAR imaging algorithm based on sparse representation has the advantages of high resolution, noise suppression and dealing with gapped data effectively. The method is based on the hypothesis that the imaging targets move smoothly. But the movement of ISAR imaging targets is usually of high maneuverability, which results in big phase error after motion compensation. Using the traditional RD imaging algorithm and the imaging algorithm based on sparse representation will make the resultant image fuzzy, and can't even be identified. This paper introduces a new rangeinstantaneous Doppler imaging algorithm based on sparse representation and time-frequency transform, which can effectively image the maneuvering target. The experimental results validate the feasibility of this approach.
\end{abstract}

\section{Introduction}

Inverse Synthetic Aperture Radar (ISAR) is an efficient remote sensing tool under all-time and all-weather circumstances. ISAR has been playing more and more important roles in many military and civilian applications including moving-target recognition, aircraft traffic control, and air/space surveillance. Because of the movement complexity of the ISAR imaging targets, parameter estimation becomes quite difficult. For spectral estimation algorithm based on the prior knowledge, it brings difficulties in practical application. So we, in general, use the robust RD algorithm. The algorithm includes two assumptions: one is that the size of the target and rotation angle is small, and the influence of migration through resolution cell (MTRC) can be neglected; the other is that target in the plane moves uniformly. But the two assumptions are always hard to meet, especially fighter plane and unmanned air vehicle of high maneuverability. During the coherent processing interval (CPI, usually second level), the speed and the direction of the rotating axis will change with time, and Doppler is time-varying. If directly utilizing traditional $\mathrm{RD}$ algorithm for imaging, the resultant image will be blurring. Therefore, in literature [1], it puts forward range-instantaneous Doppler (RID) algorithm, which mitigates image blurring. But the time-frequency resolution is limited by the length of the window, which can not meet the requirements in high resolution imaging.

The research shows that imaging methods based on sparse representation utilize the sparsity of target scene and nonlinear optimization algorithms to realize sparse representation and reconstruction of the signal, which break through the limitation of Nyquist sampling theorem and achieve the purpose of super resolution ${ }^{[2-6]}$. Therefore, the paper combines sparse representation and time-frequency transform, and constructs appropriately dictionary based on time-frequency basis, thus achieve the well focused ISAR image with super resolution. At last, this paper uses the simulation data to validate the feasibility and stableness of imaging algorithm based on sparse representation, and through simulation data with time-varying angular velocity to validate the effectiveness of the proposed method. 


\section{ISAR imaging algorithm based on Sparse Representation}

When target moves smoothly, after standard motion compensation, we can convert the target model into turntable model to deal with, shown as in Fig. 1:
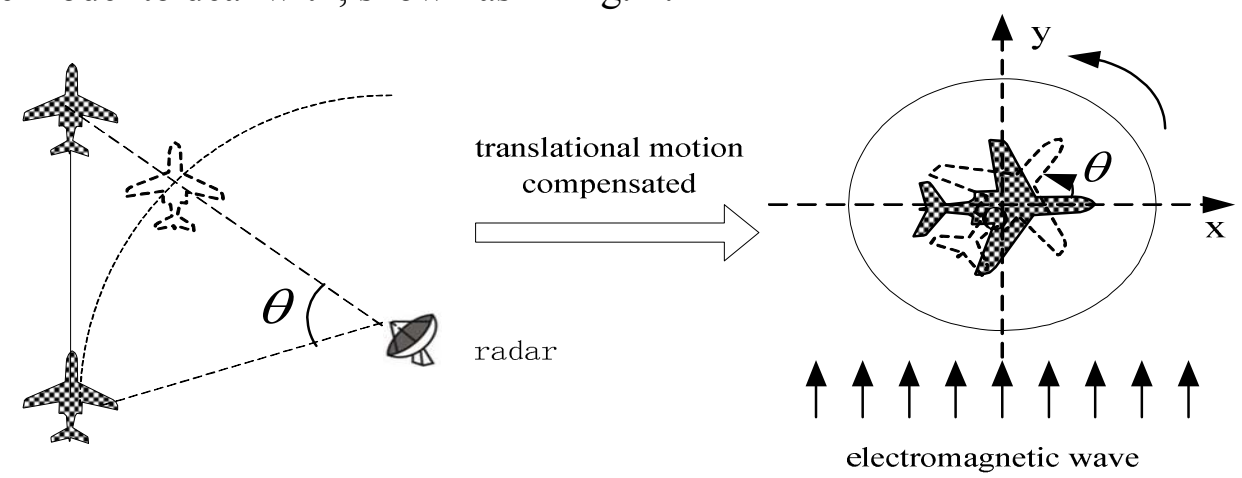

Fig. 1 ISAR turntable model

According to Geometrical Theory of Diffraction (GTD), the electromagnetic scattering of target can be considered to composition of a few of strong scattering centers in high-frequency section. After translation compensation, the target echoes can be expressed as:

$$
s(f, \theta)=\sum_{i=1}^{K} \sigma_{k} \exp \left[-j \frac{4 \pi}{c} f\left(x_{i} \cos \theta+y_{i} \sin \theta\right)\right]
$$

Where $\mathrm{f}$ is signal frequency, $\theta$ is observation angle, $x_{i}, y_{i}$ and $\sigma_{i}$ are $\mathrm{x}$ coordinate, $\mathrm{y}$ coordinate and amplitude of the ith scattering center, $K$ is the number of strong scattering centers, and $c$ is light velocity. After the range compression, we can obtain the range profiles. Because the azimuth spectrum of the single point target changes slowly, in any observation angle, its signal is monophonic. In the range bin $r_{b}$, the azimuth signal is simplified as:

$$
s\left(t, r_{b}\right)=A_{r_{b}} \delta\left(t-\frac{2 r_{b}}{c}\right) \sum_{k=1}^{K} A_{k} \exp \left(-j 2 \pi f_{k} t\right)
$$

Considering the noise and background clutter, (7) can be rewritten as:

$$
s\left(t, r_{b}\right)=A_{r_{b}} \delta\left(t-\frac{2 r_{b}}{c}\right) \sum_{k=1}^{K} A_{k} \exp \left(-j 2 \pi f_{k} t\right)+n
$$

Where $\mathrm{n}$ is the noise, and $\mathrm{K}$ is the number of strong scattering centers. In the frequency domain, the number of effective scattering centers is small, the other is zero. This is similar to the sparse presentation model. Fourier basis space is widely applied in all kinds of sparse representation algorithms. The dictionary $\Psi$ based on Fourier basis is expressed as follow:

$$
\Psi=\left[\begin{array}{cccc}
e^{-j \omega_{0} t_{0}} & e^{-j \omega_{1} t_{0}} & \cdots & e^{-j \omega_{M-1} t_{0}} \\
e^{-j \omega_{0} t_{1}} & e^{-j \omega_{1} t_{1}} & \cdots & e^{-j \omega_{M-1} t_{1}} \\
\vdots & \vdots & \vdots & \vdots \\
e^{-j \omega_{0} t_{N-1}} & e^{-j \omega_{1} t_{N-1}} & \cdots & e^{-j \omega_{M-1} t_{N-1}}
\end{array}\right]
$$

Where $\left[t_{0}, t_{1}, t_{2}, \cdots, t_{N-1}\right]^{T}$ is the azimuth time series, and $\left[\omega_{0}, \omega_{1}, \omega_{2}, \cdots, \omega_{N-1}\right]$ is the discrete Doppler series. Then, (8) is rewritten as:

$$
s(t)=\Psi \theta+n
$$


Where $\theta$ denotes the vector whose nonzero components correspond to the complex amplitudes of the strong scattering centers. In the practical situation, the number of equation in (5) is less than the unknown. To overcome the uncertain of this equation and acquire the accurate $\theta$ is quite difficult .We can obtain reliable $\theta$ by solving a optimization problem as

$$
\min \left(\left\|\theta^{\prime}\right\|_{0}\right) \text {, s.t. }\left\|s(t)-\Psi \theta^{\prime}\right\|_{2} \leq \varepsilon \quad\left(l_{0} \text { problem }\right)
$$

Where $\theta^{\prime}$ is the estimated scattering center amplitudes, $\varepsilon$ is noise level. $l_{0}$ norm is non-convex, and minimizing $l_{0}$ norm will cause the chaotic optimization problems, and it is sensitive to noise. So a number of approximations have been considered in the literature, including $l_{1}$-norm and $l_{p}$-norm approximations(with $p<1$ ) which can be solved using continuous optimization methods:

$$
\begin{array}{cr}
\min \left(\left\|\theta^{\prime}\right\|_{1}\right), \quad \text { s.t. }\left\|s(t)-\Psi \theta^{\prime}\right\|_{2} \leq \varepsilon & \left(l_{1} \text { problem }\right) \\
\min \left(\left\|\theta^{\prime}\right\|_{p}^{p}\right) \text {, s.t. }\left\|s(t)-\Psi \theta^{\prime}\right\|_{2} \leq \varepsilon, p<1 & \left(l_{p} \text { problem }\right)
\end{array}
$$

The $l_{1}$ problem can be solved efficiently by matching pursuit and its variants, and recently, convex optimization $\left(l_{1}\right)$. The $l_{p}$ problem is non-convex, but a locally optimal solution can be found by local optimization; for some applications the resulting local minima are observed to give excellent approximations. Although the exact notion of sparsity (expressed by an $l_{0}$-norm ) is replaced by $l_{1}$ and $l_{p}$ norms in the latter two, it can be shown that under some conditions these relaxations solve the original problem exactly.

In addition, the performance of the imaging algorithm based on sparse representation is closely related to the parameter $\varepsilon$. If the parameter is set much smaller, the resultant image suffer from artifacts induced by noise; if it is much bigger, a part of the target scattering centers is treated as noise to suppress. So calculating accurate noise level $\varepsilon$ plays a vital role to the final imaging performance. Using energy threshold to select range noise cell, if the energy of a certain range cell is less than the set threshold, it is regarded as the range bin containing only noise. Using these range noise bins estimate noise level $\varepsilon$. The threshold can be expressed as follow ${ }^{[4]}$ :

$$
\text { thres }=E_{m}+\left[\sum_{i=1}^{I} \frac{\left(E_{i}-E_{m}\right)^{2}}{I-1}\right]^{1 / 2}
$$

Where $E_{i}$ is the energy of the ith range cell, $E_{m}$ is the average energy of the whole range cells, and $I$ is the number of the whole range cell. Fig. 2 is that the range bins containing only noise are separated by the energy threshold using the real data of Boeing-727.

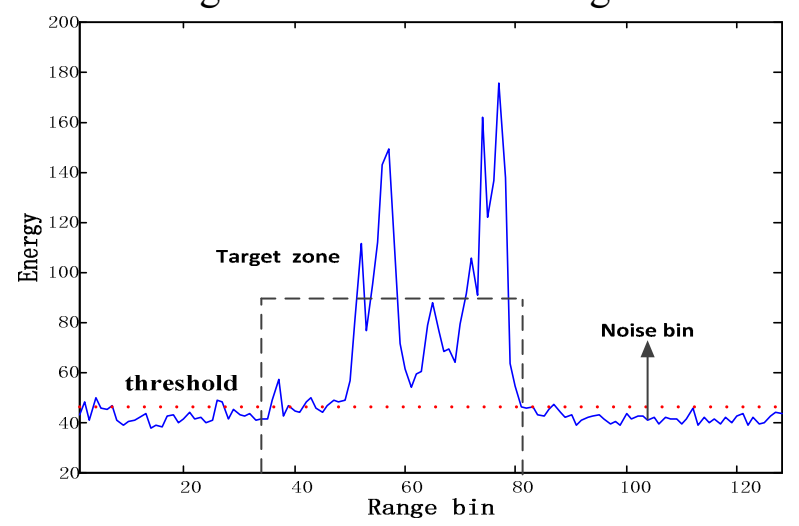

Fig. 2 Discrimination of target from noise in the coarse range profile

\section{ISAR imaging algorithm based on sparse representation and time-frequency transform}

The previous section introduces the ISAR imaging algorithm based on sparse representation, under the situation that the target moves smoothly, so the resultant image is well focused. However, ISAR imaging targets are usually maneuvering, during CPI (usually second level), angular speed and the 
direction of rotation change with time, which will also lead to the target size and shape in the projection plane change with the observation time. After the motion compensation, Because of the imaging target involving pitch, roll, and yaw, we can use synthetic vector $\boldsymbol{\Omega}$ as the rotation axis. The direction and the value of $\boldsymbol{\Omega}$ are time-varying during CPI, thus imaging projection plane and the scale of azimuth change with time. For any moment of corresponding $\boldsymbol{\Omega}$, it has corresponding projection plane. So, as long as depending on the Doppler information of every scattering center of any moment, we can obtain range-instantaneous Doppler image in the moment. Using the time-frequency transform instead of the Fourier transform, we can obtain of range-instantaneous Doppler image of different time moment. This is RID imaging algorithm proposed in the article ${ }^{[1]}$.

RID algorithm can effectively solve image blurring brought by time-varying Doppler, but the resolution of this algorithm is limited by the length of the window, which can not meet the requirements in high resolution imaging. In order to solve the limitations of the RID algorithm, and the ISAR imaging algorithm based on sparse presentation that can't deal with time-varying data, so we introduce a kind of imaging algorithm which joints the two algorithms. Making fully use of the idea of time-frequency analysis to construct appropriate dictionary based on time-frequency basis, and then utilizing optimization reconstruction algorithm for imaging, thus we can effectively solve imaging blurring caused by Doppler time-varying.

The proposed imaging method uses time-frequency basis instead of Fourier basis. In this paper, we use the time-frequency basis based on Gabor transform to construct the following dictionary:

$$
\begin{gathered}
\Psi_{p}^{T F}(:, m)=\frac{1}{\pi^{1 / 4} \sqrt{\sigma}} \exp \left\{\frac{-\left(t-t_{p}\right)^{2}}{2 \sigma^{2}}\right\} \exp \left(-j w_{m}\left(t-t_{p}\right)\right) \\
m=1,2, \cdots, M-1, M
\end{gathered}
$$

Where $\mathrm{p}$ is time frame, $w_{m}$ is the Doppler frequency, and $\mathrm{M}$ is the number of frequency bins. Because Doppler-shifts is not obvious from a sampling to the next sampling, so the $\mathrm{p}$ value can be selected smaller to reduce the computational complexity.

Then, Eq. 4 is transformed into the following optimization problem:

$$
\begin{gathered}
\min \left(\left\|\theta_{p}{ }^{\prime}\right\|_{1}\right), \quad \text { s.t. }\left\|S(t)-\Psi_{p}^{T F} \theta_{p}^{\prime}\right\|_{2} \leq \varepsilon, \\
p=1,2,3 \cdots, P
\end{gathered}
$$

Where $\theta_{p}^{\prime}$ is estimation of Doppler in a certain time moment $p$. Then we can obtain high resolution well-focused ISAR image sequence by solving the optimization problem.

In principle, any time-frequency basis can be used in this place. But a time-frequency basis should meet the following requirements: 1 . high resolution in both time and frequency domain; 2 . it can accurately reflect the signal instantaneous frequency. This paper only introduce imaging algorithm, and the time-frequency basis is not the focus of the paper. So we select the commonly used time-frequency basis based on Gabor transformation in the following experiments.

The whole algorithm procedure is as followed:

(1) motion compensation and range compression;

(2) calculate the noise level $\varepsilon$;

(3) construct appropriate dictionary based on time-frequency basis and use optimization reconstruction algorithm to solve the equation (11);

(4) produce well focused ISAR image sequence $I(p),(p=1,2, \cdots, P-1, P)$ with high resolution. Where $I(p)$ is instantaneous Doppler ISAR image in the moment $\mathrm{p}$.

\section{The experiment results and analysis}

The imaging performance of ISAR imaging algorithm only based on sparse representation. Simulation data from Boeing727 airplane is used in the experiment ${ }^{[7]}$. The radar sends 64 bursts, each having 256 modulated pulses. The frequency of the starting pulse is $f_{0}=9 \mathrm{GHz}$, and the total 
frequency bandwidth is $B=150 \mathrm{MHz}$. The pulse repetition frequency is $20 \mathrm{KHz}$. Fig. 3(a)-(c) shows the reconstructed image from the traditional RD method and imaging algorithm based on sparse representation from the partial data and full data respectively. The reconstruction was done using $30 \%$ and $70 \%$ random slow-time under-sampled phase histories respectively. As can be seen from Fig. 3 that sparse representation imaging method provides a good reconstruction compared to the traditional RD method .Compared with traditional RD imaging algorithm, imaging algorithm based on sparse representation has better performance with higher resolution, and deals with the gapped data effectively.
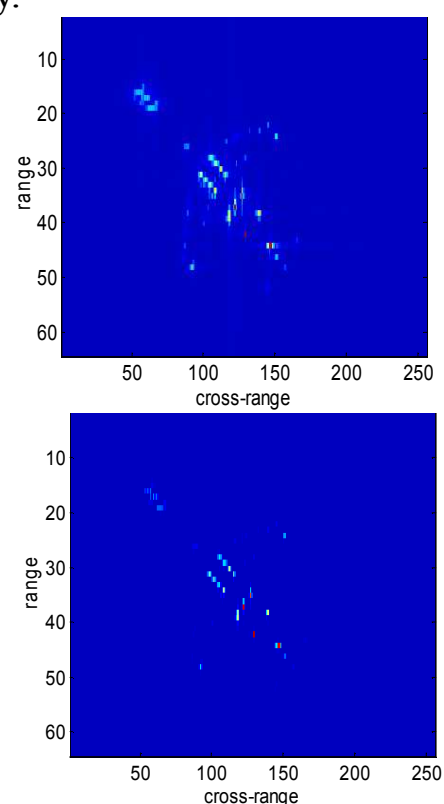

(a)
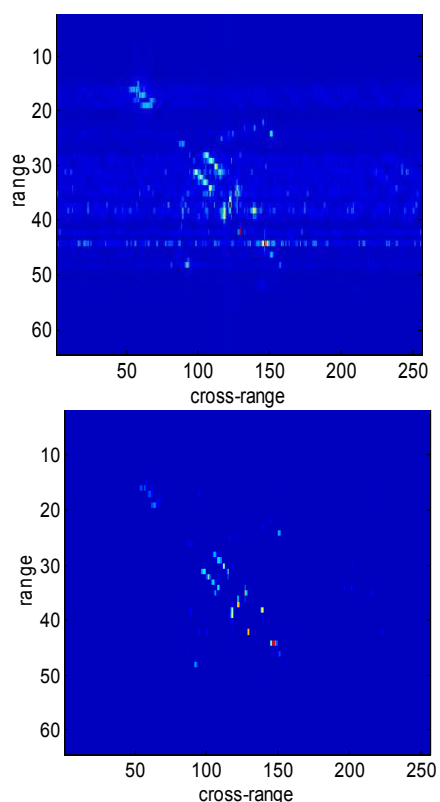

(b)
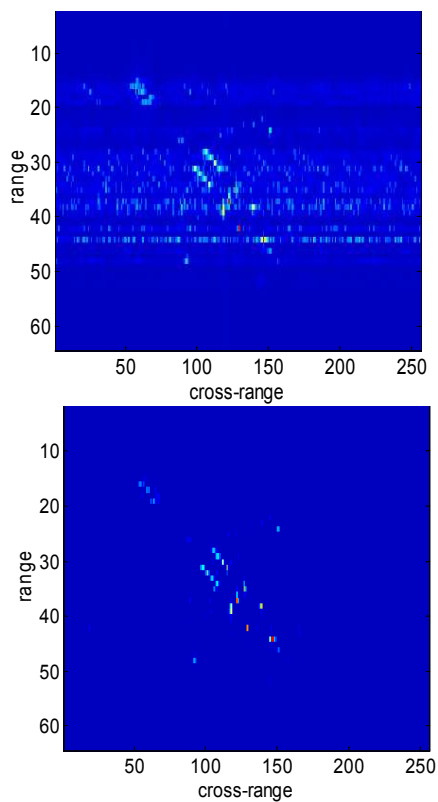

(c)

Fig. 3 Imaging performance via RD algorithm(top row) and sparse representation imaging algorithm(second row) of different gapped data. (a) the full data (b) $70 \%$ used (c) $30 \%$ used

The imaging performance and comparison of the proposed method. In order to validate the proposed method dealing with Doppler variant data, the simulated echo data of Boeing 727 is used ${ }^{[7]}$. The radar sends 256 bursts, each having 64 modulated pulses. The frequency of the starting pulse is $f_{o}=9 \mathrm{GHz}$, and the total frequency bandwidth is $B=150 \mathrm{MHz}$. The pulse repetition frequency is 20 $\mathrm{KHz}$. While constructing the simulation echo, the rotation angular velocity is not constant but time-varying. Using RD algorithm and the ISAR imaging algorithm based on sparse representation directly, the resultant image becomes blurring, as shown in Fig. 4(a-b). Because of the time-varying of angular velocity, after the motion compensation, the rest of the phase error is still quite big, so the resultant image is not focused but blurring. Fig. 4(c) is the result of the imaging using RID algorithm, and there exists no blurring. But the resolution is not as good as the method proposed in the paper, as shown in Fig. 4(d).

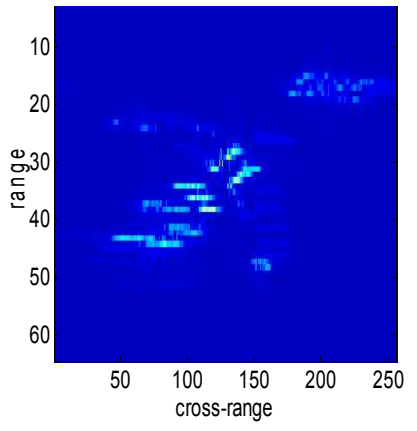

(a)

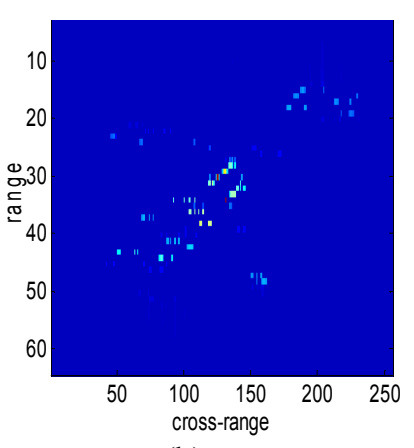

(b)

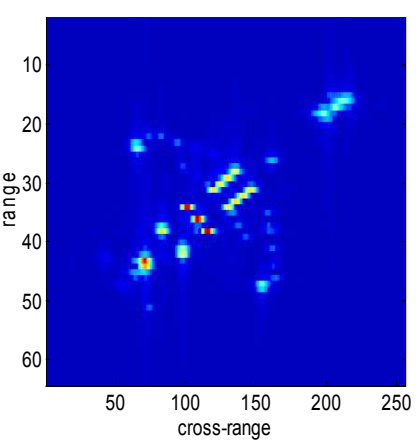

(c)

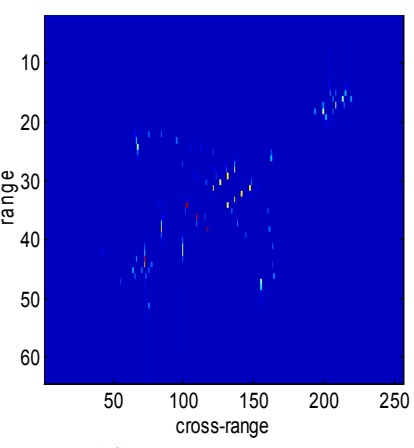

(d)

Fig. 4 The imaging performance of the four imaging algorithms for the time-varying simulation data (a) RD (b) sparse presentation imaging algorithm (c) RID algorithm (d) the proposed method 


\section{Summary}

Using the traditional imaging method based on FFT and the imaging algorithm based on sparse representation directly to image the maneuvering target, the resultant image is blurring. So this paper introduces an ISAR imaging algorithm combining sparse representation with time-frequency transform. The algorithm constructs appropriate dictionary based on time-frequency basis and use the optimization reconstruction algorithm to image. Finally, the processing results using the simulation data show that the proposed method effectively deals with time-varying data, and there exists no image blurring. Compared with RID algorithm, the image resolution is greatly improved.

\section{Acknowledgements}

This research was supported by the National Natural Science Foundation of China under Grant 61073106, Aerospace Science Foundation under Grant 2011ZC53042, and graduate starting seed fund of Northwestern Polytechnical University under Grant Z2012094.

\section{References}

[1] Z. Bao, G. Y. Wang, L. Luo, Range-Instantaneous Doppler Imaging in ISAR, Acta Electronica Sinica[J], 1998, Vol. 26(12): 79-83.

[2] S. Samadi, M. Cetin, M.A. Masnadi-Shirazi,Sparse representation-based synthetic aperture radar imaging[J]. IEEE Proc.Radar Sonar Navig., 2011, 5 (2): 182 -193.

[3] K. R. Varshney, M. Çetin, and J. W. Fisher, et al., Sparse Representation in Structured Dictionaries with Application to Synthetic Aperture Radar[J]. IEEE Trans on Signal Processing. 2008, Vol. 56(8):3548-3561.

[4] Zhang L, Xing M D, and Qui C W, et al.. Resolution Enhancement for Inversed Synthetic Aperture Radar Imaging Under Low SNR via Improved Compressive Sensing. IEEE Transactions on Geoscience and Remote Sensing, 2010, 48(10): 3824-3838.

[5] C. D. Austin, E. Ertin, and R. L. Moses. Sparse Signal Methods for 3-D Radar Imaging[J]. IEEE Journal of Selected Topics in Siganl Processing, JUNE 2011,VOL. 5, NO. 3, 408-423

[6] H. X. Wang, Y. Liang, and M. D. Xing, et al.. Sparse Signal Representation-Based Space Debris Imaging[J]. Journal of Astronautics, 2011, Vol. 32, No. 10, 2194-2199.

[7] V. C. Chen and H. Ling. Time-Frequency Transforms for Radar Imaging and Signal Analysis. Norwood, MA: Artech House, 2002. 